\title{
COUNTERION EFFECTS ON THE STRUCTURE OF LANGMUIR-BLODGETT FILMS OF A BENZOTHIAZOLIUM DYE
}

\author{
G.J. Ashwell*, G. Jefferies*, D.G. Hamilton and T. Handa ${ }^{\dagger}$ \\ Centre for Molecular Electronics, Cranfield University, Cranfield MK43 OAL, UK \\ (Received May 5, 1995; in final form June 12, 1995)
}

Dedicated to Professor Krzysztof Pigon on the occasion of his 70th birthday ${ }^{\ddagger}$

Langmuir-Blodgett films of salts of the two-legged cationic dye, $E$ - $N$-hexadecyl-2-[2-(4-docosylaminophenyl)ethenyl]benzothiazolium, are $Z$-type. The molecular configuration within the Langmuir-Blodgett film is either "U-shaped" or "stretched" depending upon the nature of the anion, the thickness per layer being $35.1 \AA$ for the octadecylsulphate and $54.1 \AA$ for the iodide. For the Langmuir-Blodgett monolayers the principal components of the second order susceptibility $\left(\chi_{z z z}^{(2)}\right)$ are $70 \mathrm{pm} \mathrm{V}^{-1}$ and $34 \mathrm{pm} \mathrm{V}^{-1}$ respectively at $532 \mathrm{~nm}$. In the case of the octadecylsulphate salt it was possible to fabricate thick multilayer Langmuir-Blodgett films; the second harmonic intensity increased with the number of $Z$-type layers, albeit subquadratically, and this may be ascribed to a progressive variation in the chromophore tilt angle.

PACS numbers: 73.20.Mf, 81.15.Lm

\section{Introduction}

Long-range order within molecular films is a prerequisite for a diverse range of solid state applications. For example, both pyroelectricity and second harmonic generation (SIIG) require the structure to be non-centrosymmetric. In addition, sensing strategies require preferred orientation of the response elements to both reduce diffusive processes and facilitate analy te binding mechanisms. Thus, the alignment and packing of molecular materials is of paramount importance when considering the deposition characteristics of a particular species. The Langmuir-Blodgett

\footnotetext{
*To whom correspondence should be addressed.

†Permanent address: TDK Corporation, TDK Chikumagawa 1st Technical Centre, 462-1 Otai, Saku-shi, Nagano 389-02, Japan.

I Submitted on invitation of the Institute of Physical and Theoretical Chemistry, Technical University of Wrocław, Wrocław, Poland.
} 
(LB) technique provides an effective tool whereby such ordering can be controlled at the molecular level.

Successive deposition of floating Langmuir films can be controlled to produce LB multilayers with sustained $Y$-type (centrosymmetric) and $X$-type or $Z$-type (non-centrosymmetric) packing arrangements. Asymmetry can be induced by tailoring the materials to force $X$-type or $Z$-type deposition [1-7] or be acquired by interleaving $Y$-type layers with optically inactive spacers [8-12]. For homomolecular films in excess of 100 layers such long range non-centrosymmetry has only been realised for a modicum of materials, namely 2-docosylamino-5-nitropyridine (DCANP) [13], a quinolinium zwitterion $\left(\mathrm{C}_{16} \mathrm{H}_{33}-\mathrm{Q} 3 \mathrm{CNQ}\right)$ [4] and a series of unconventional LB dyes comprising a chromophore with hydrophobic chains at opposing ends [5-7].

The nature of the counterion can also affect the film properties and packing arrangement [11-16]. For example, the use of octadecylsulphate instead of bromide has dramatically improved the SHG from stilbazolium dyes [17] and the presence of primary amines or metal ions $\left(\mathrm{Co}^{2+}\right.$ or $\left.\mathrm{Ba}^{2+}\right)$ has been shown to control the extent of $J$-aggregation in merocyanine dyes [18]. In this study we investigated the effect of iodide and octadecylsulphate counterions on the film-forming and nonlinear optical properties of a benzothiazolium dye (Fig. 1). The smaller iodide

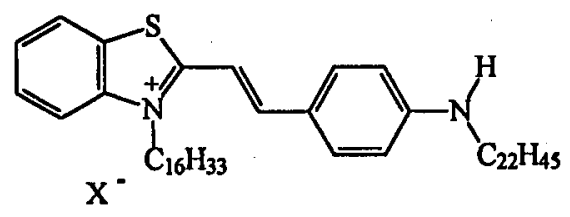

Fig. 1. Molecular structure of $E$ - $N$-hexadecyl-2-[2-(4-docosylaminophenyl)etheny]]benzothiazolium $\mathrm{X}^{-}$, where $\mathrm{X}^{-}$is either iodide or octadecylsulphate.

anion gave rise to an extended molecular configuration in which the hydrophobic chains are oriented in opposite directions from the chromophore. By contrast, the octadecylsulphate anion resulted in an altered packing motif in which the molecule adopts a "U-shaped" configuration whereby all of the alkyl chains point in the same direction.

\section{Langmuir-Blodgett films}

The iodide and octadecylsulphate salts of the benzothiazolium dye were spread, from chloroform and methanol/chloroform solutions respectively, at concentrations of $0.1 \mathrm{mg} \mathrm{ml}^{-1}$ onto the pure water subphase of an alternate layer LB trough (Nima Technology). The films were compressed at $0.5 \mathrm{~cm}^{2} \mathrm{~s}^{-1}$ to a surface pressure of $35 \mathrm{mN} \mathrm{m}^{-1}$ and deposited, on the upstroke, onto hydrophilically treated glass substrates and silver coated slides at a rate of $0.08 \mathrm{~mm} \mathrm{~s}^{-1}$.

Isotherms of the iodide and octadecylsulphate salts were obtained at $c a$. $20^{\circ} \mathrm{C}$ (Fig. 2). At zero pressure both isotherms exhibit similar molecular areas of $110 \AA^{2}$ molecule ${ }^{-1}$ suggesting a "U-shaped" conformation with the chromophore 


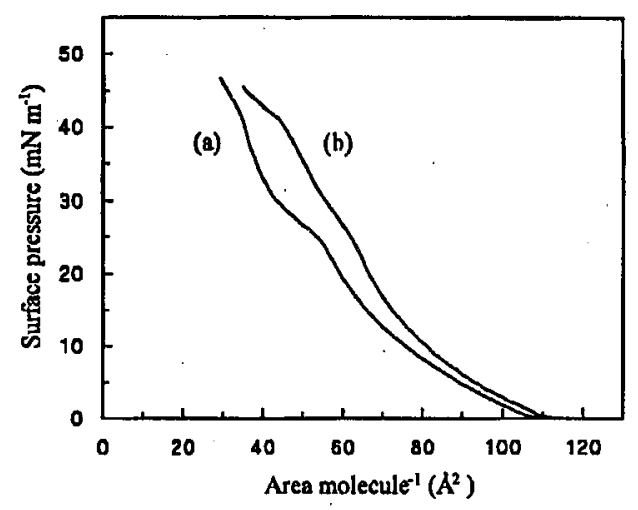

Fig. 2. Surface pressure versus area isotherm of the benzothiazolium dye spread from $0.1 \mathrm{mg} \mathrm{ml} \mathrm{m}^{-1}$ solutions at $c a .20^{\circ} \mathrm{C}$ and compressed at $0.5 \mathrm{~cm}^{2} \mathrm{~s}^{-1}$, where (a) is the iodide salt and (b) the octadecylsulphate salt.

adjacent to the water surface. However, just prior to collapse the areas reduce to $29 \AA^{2}$ molecule $^{-1}$ with iodide and $44 \AA^{2}$ molecule $^{-1}$ with octadecylsulphate suggesting an altered configuration. Based on the surface plasmon resonance (SPR) analysis of deposited films of the iodide salt there is evidence that, in this case, the molecule has a stretched motif. Although the existence of a hydrophobic chain juxtaposed to the interface is unusual, such alignments have previously been confirmed by neutron reflectivity studies on Langmuir films of $Z-\beta$ - $(N$-decyl-4-quinolinium)- $\alpha$-cyano-4-styryldicyanomethanide $\left(\mathrm{C}_{10} \mathrm{H}_{21}\right.$-Q3CNQ) which forms antiparallel dimers at the air-water interface [4].

\section{Surface plasmon resonance}

SPR studies were carried out using an ATR geometry in the Kretschmann configuration [19]. An approximately $460 \AA$ thick silver film was vacuum deposited onto a clean glass substrate and index matched to one face of an equilateral BK7 glass prism. Reflectivity data were obtained as a function of incidence angle using a $p$-polarised incident beam from a $532 \mathrm{~nm} \mathrm{Nd:YAG} \mathrm{laser.}$

SPR reflectivity data were first corrected for reflections at the entrance and exit faces of the prism and analysed by comparison to the Fresnel reflection formulae by a method previously described $[4,20,21]$. The thickness (typically $c a$. $460 \AA)$ and the real $\left(\varepsilon_{\mathrm{r}}\right)$ and imaginary $\left(\varepsilon_{\mathrm{i}}\right)$ parts of the dielectric permittivity were initially obtained for silver $\left(\varepsilon_{\mathrm{r}}=-10.8 \pm 0.1, \varepsilon_{\mathrm{i}}=0.51 \pm 0.03\right.$ at $\left.532 \mathrm{~nm}\right)$ and these values fixed in the subsequent analysis of the reflection data for overlaid LB films. This gave rise to curves of the type illustrated in Fig. 3. The real and imaginary parts of the dielectric permittivity were similar for the two salts, the values being 2.98 and 0.426 respectively for the iodide and 2.62 and 0.452 for the octadecylsulphate. Interestingly the thicknesses are markedly different, being $54.1 \AA$ layer $^{-1}$ for the iodide and $35.1 \AA$ layer $^{-1}$ for the octadecylsulphate. The former complies with a fully stretched motif and may be compared with the van der Waals length of 


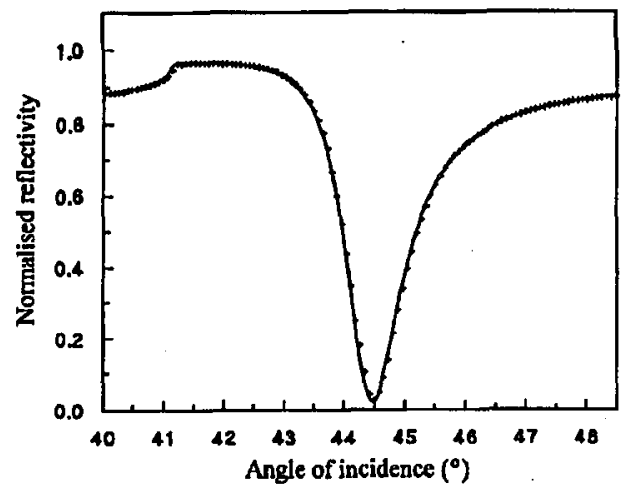

Fig. 3. Normalised theoretical (solid line) and experimental (crosses) SPR reflectivity data for a monolayer of a 1:1 molar ratio of benzothiazolium iodide and sodium octadecylsulphate. The water soluble sodium iodide, formed by metathesis, dissolves into the subphase.

$57 \AA$. However, the thickness for the octadecylsulphate salt suggests a "U-shaped" molecular configuration similar to that proposed for the Langmuir isotherm at low pressures. Thus, the long chain anion alters the architecture of the deposited film.

\section{Nonlinear optical effects}

The measurements of SHG were carried out in transmission using a $1064 \mathrm{~nm}$ $\mathrm{Nd}$ :YAG laser incident at $45^{\circ}$ to the normal of the LB film. The polarisation dependence of the second harmonic intensity, $I_{2 \omega}(p \rightarrow p) / I_{2 \omega}(s \rightarrow p)$, was analysed for the monolayer using the method of Kajikawa et al. [22]. This gave rise to chromophore tilt angles, from the normal to the substrate, of $40^{\circ}$ for the iodide and $32^{\circ}$ for the octadecylsulphate. These values, the thicknesses and refractive indices from SPR, and the SH intensity data provide principal second order susceptibility components of $\chi_{z z z}^{(2)}=34 \mathrm{pm} \mathrm{V}-1$ for monolayer films of the iodide and $\chi_{z z z}^{(2)}=$ $70 \mathrm{pm} \mathrm{V}^{-1}$. for monolayer films of the octadecylsulphate. This indicates that the use of a long chain anion results in an alternative packing structure giving rise to enhanced $\chi^{(2)}$ effects.

$Z$-type multilayer films of the octadecylsulphate salt have been successfully deposited to 200 layers, although similar results could not be obtained for the iodide analogue. The SH intensity increased with the number of layers (Fig. 4) indicating a $Z$-type structural arrangement, although this did not vary according to theory (i.e. $I_{2 \omega}=I_{2 \omega(1)} N^{2}$, where $N$ is the total number of LB layers). The curvature for thicker films may be due, in part, to absorbance of the SH intensity although the dominant effect is more likely to be the variation in the tilt of the chromophores. The polarisation dependence indicates a change in tilt angle from ca. $32^{\circ}$ for the monolayer to an average value of ca. $79^{\circ}$ for the 200 layer film (Fig. 5). 


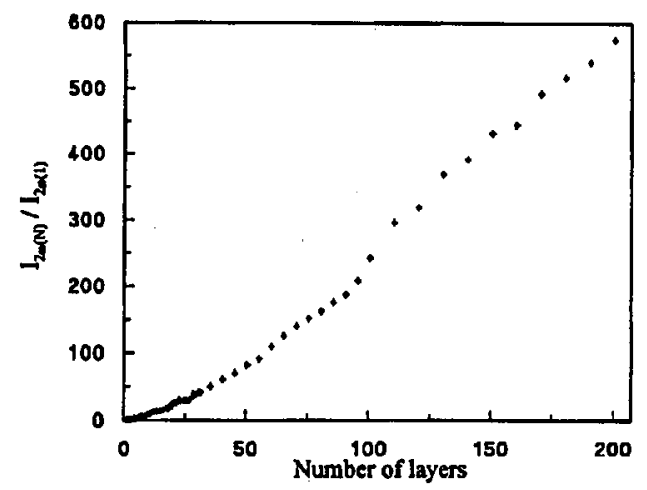

Fig. 4. Ratio of film to monolayer second harmonic intensity $\left(I_{2 \omega(N)} / I_{2 \omega(1)}\right)$ with increasing number of LB layers showing the subquadratic behaviour of the benzothiazolium octadecylsulphate salt.

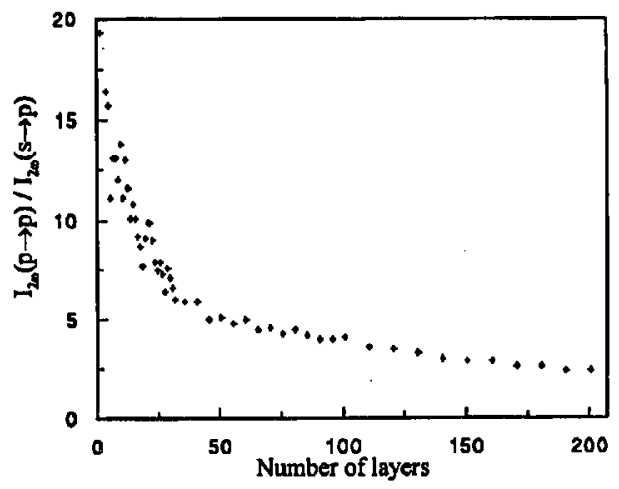

Fig. 5. Variation in the polarisation dependence $\left[I_{2 \omega}(p \rightarrow p) / I_{2 \omega}(s \rightarrow p)\right]$ with increasing number of LB layers of benzothiazolium octadecylsulphate.

\section{Conclusion}

The thin film characteristics of a two-legged benzothiazolium dye have been investigated to ascertain the impact of structurally disparate anions. The molecular configuration is dependent upon the type of anion and may adopt either a "stretched" or "U-shaped" arrangement within the film. Z-type multilayer structures have successfully been deposited to 200 LB layers, albeit with subquadratic enhancement, though this may be attributable to the variation in tilt angle as the film thickness increases.

\section{Acknowledgments}

We thank the EPSRC (UK) and TDK (Japan) for financial support of the nonlinear optics programme at Cranfield and for providing studentships to G.J. and T.H. 


\section{References}

[1] O.A. Aksipetrov, N.N. Akhmediev, I.M. Baranova, E.D. Mishina, V.R. Novak, Sov. Tech. Phys. Lett. 11, 249 (1985).

[2] R. Popovitz-Biro, K. Hill, E.M. Landau, M. Lahav, J. Leiserowitz, J. Sagiv, J. Am. Chem. Soc. 110, 2672 (1988).

[3] T. Richardson, G.G. Roberts, M.E.C. Polywka, S.G. Davies, Thin Solid Films 160, 231 (1988).

[4] G.J. Ashwell, G. Jefferies, E.J.C. Dawnay, A.P. Kuczynski, D.E. Lynch, Y. Gongda, D.G. Bucknall, J. Mater. Chem., in press.

[5] G.J. Ashwell, P.D. Jackson, W.A. Crossland, Nature 368, 438 (1994).

[6] G.J. Ashwell, Y. Gongda, D. Lochun, P.D. Jackson, Polym. Prep. 35, 185 (1994).

[7] G.J. Ashwell, T. Handa, G. Jefferies, D.G. Hamilton, J. Colloids Surf. Sci., in press.

[8] G.J. Ashwell, E.J.C. Dawnay, A.P. Kuczynski, P.J. Martin, SPIE - Int. Soc. Opt. Eng. 1361, 589 (1991).

[9] G.J. Ashwell, P.D. Jackson, D. Lochun, P.A. Thompson, W.A. Crossland, G.S. Bahra, C.R. Brown, C. Jasper, Proc. R. Soc. Lond. A 445, 385 (1994).

[10] M. Era, K. Nakamura, T. Tsutsui, S. Saito, H. Niino, K. Takehara, K. Isomura, H. Tanaguchi, Jpn. J. Appl. Phys. 29, L2261 (1990).

[11] P. Hodge, Z. Ali-Adib, D. West, T.A. King, Macromolecules 26, 1789 (1993).

[12] T.L. Penner, N.J. Armstrong, C.S. Willand, J.S. Schildkraut, D.R. Robello, SPIE - Int. Soc. Opt. Eng. 1360, 377 (1991).

[13] G. Decher, B. Tieke, C. Bosshard, P. Günter, J. Chem. Com. Soc., Chem. Commun. 933 (1988).

[14] M.C.J. Young, R. Jones, R.H. Tredgold, W.X. Lu, Z. Ali-Adib, P. Hodge, F. Abbassi, Thin Solid Films 182, 319 (1989).

[15] X. Liu, L. Liu, Z. Chen, X. Lu, J. Zheng, W. Wang, Thin Solid Films 219, 221 (1992).

[16] M.K. DeArmond, H. Samha, O. Dvorak, Langmuir 10, 343 (1994).

[17] G.J. Ashwell, R.C. Hargreaves, C.E. Baldwin, G.S. Bahra, C.R. Brown, Nature 357, 393 (1992).

[18] T. Kawaguchi, K. Iwata, Thin Solid Films 180, 235 (1989).

[19] E. Kretschmann, Z. Phys. 241, 313 (1971).

[20] W.L. Barnes, J.R. Sambles, Surf. Sci. 177, 399 (1986).

[21] W.L. Barnes, J.R. Sambles, Surf. Sci. 183, 189 (1987).

[22] K. Kajikawa, K. Kigata, H. Takezoe, A. Fukuda, Mol. Cryst. Liq. Cryst. A 182, 91 (1990). 